\title{
Strength and Power Improvements in Athlete and Non-Athletes During Intensive Eating Disorder Treatment
}

\author{
Laura K. Fewell \\ University of Missouri-St. Louis \\ Riley Nickols and Amanda Schlitzer Tierney \\ McCallum Place Eating Disorder Treatment Centers
}

\author{
Cheri A. Levinson \\ University of Louisville
}

\begin{abstract}
Understanding the unique needs of athletes who undergo eating disorder (ED) treatment is sorely needed. This study explores changes of strength and power in athlete $(n=21)$ and non-athlete $(n=36)$ patients from intake to discharge. Maximal oxygen consumption, vertical jump, push-ups, hand grip strength, and body mass index (in anorexia nervosa; AN) were measured among treatment center patients. The number of push-ups and hand grip strength were significantly improved upon discharge in the full sample $(p s<.005)$ and in AN only $(p s<.001)$. Body mass index was also significantly higher in AN $(p<.001)$. Maximal oxygen consumption and vertical jump did not significantly improve between admission and discharge in either group ( $p s>.40$ ). This study is the first to investigate measures of strength in athletes engaged in intensive eating disorder treatment and indicates the need to address the psychological mindset around physical activity using exercise education as part of a comprehensive program. Recommendations for incorporating exercise into an intensive ED treatment center are also provided.
\end{abstract}

Keywords: athlete, eating disorder, sport, strength, treatment

Eating disorders (EDs) are serious and life impairing illnesses (Harris \& Barraclough, 1998) and are present in approximately $4.6 \%$ of the population (Sundgot-Borgen \& Torstveit, 2004). Some studies have found that EDs are even

Fewell is with the Dept. of Psychological Sciences at University of Missouri-St. Louis. Nickols and Schlitzer Tierney are with The Victory Program, McCallum Place Eating Disorder Treatment Centers, St. Louis, MO. Levinson is with the Dept. of Psychological and Brain Sciences at University of Louisville, Louisville, KW. Address author correspondence to Cheri A. Levinson at cheri.levinson@ louisville.edu. 
more common among athletes, with rates as high as $13.5 \%$ (Sundgot-Borgen, 1993) and disordered eating as high as $25.5 \%$ (Greenleaf, Petrie, Carter, \& Reel, 2009). However, treatment providers and programs may fail to tailor treatment approaches to meet the unique needs of athletes with EDs. For example, possessing a commitment to rigorous sport training is a core feature of highlevel athleticism. Yet exercise is often abused among those with EDs (Beumont, Arthur, Russell, \& Touyz, 1994; Katz, 1996) and intensive treatment centers have historically avoided incorporating exercise into treatment to mitigate potential liability concerns and to prevent undermining weight restoration goals. A primary motivation for many athletes in ED treatment recovery is to be able to return to their sport (Arthur-Cameselle \& Quatromoni, 2014) and athletes and coaches believe that training is key to attaining or maintaining enhanced sport performance (Bompa, 1983). Therefore, intensive ED treatment could serve as an opportune setting to provide necessary monitoring and support for athletes with EDs by focusing on exercise and training.

Recent literature has advocated for the incorporation of exercise within ED treatments and suggests that exercise can be a positive contribution to ED treatments if incorporated appropriately (Carei, Fyfe-Johnson, Breuner, \& Brown, 2010; Cook et al., 2016; Reel, 2015; Reel \& Voelker, 2012; Sherman, Cherkin, Erro, Miglioretti, \& Deyo, 2005). Specifically, intensive ED treatment programs should include "prescribed" exercise that is designed by the treatment team for the client's individual needs. Further, appropriate training regimens and exercise goals for athletes should be incorporated into intensive ED treatment programs.

\section{Athletes and Intensive ED Treatment}

Intensive ED treatment centers, which include inpatient (IP), residential, and partial hospitalization programming (PHP), typically have similar goals of normalizing disordered eating behaviors, restoring weight when indicated, and improving irrational belief systems around eating and weight (Olmsted et al., 2007). The role of sport for the athlete (i.e., return to sport, reintegration of sport specific training, cultivating a more balanced relationship with sport) is not typically amalgamated or supported within intensive ED treatment centers. Current guidelines for treating athletes with EDs focus on the outpatient level of care and recommend a multidisciplinary team comprised of a physician, sports dietitian, mental health professional, and athletic trainer (Joy et al., 2014; Joy, Kussman, \& Nattiv, 2016; Mountjoy et al., 2014). Multiple studies have also demonstrated effective prevention efforts to reduce disordered eating and prevent EDs in athletes (Bar, Cassin, \& Dionne, 2016; Becker, McDaniel, Bull, Powell, \& McIntyre, 2012; Martinsen et al., 2014; Torres-McGehee et al., 2011), but few, if any, have provided recommendations for treating athletes in an intensive level of care.

Much of the treatment recommendations for severely impaired ED athlete patients focus on improving physical health and return to sport. Baum (2006) proposed several strategies for treating the male athlete including: prioritizing a healthy weight and metabolic balance; utilizing psychopharmacological treatments when warranted; providing psychotherapy, which may include both the biological and sport family; and providing vocational rehabilitation if necessary. Although this study does not account for the role of exercise in intensive ED treatment, others 
(e.g., Plateau, Arcelus, Leung, \& Meyer, 2017) suggest that it would be beneficial to address compulsive exercise with athletes in ED treatment. Moreover, focusing on an athlete's relationship to sport has not been demonstrated to be a part of intensive ED care. Indeed, after interviewing 13 female athletes with EDs, Plateau et al. (2017) discovered that athlete patients felt out of place and lacked motivation to engage in treatment due to perceived irrelevance within the treatment programs. The athletes also reported difficulty refraining from exercise and concern over transitioning back to sport. The authors recommended that treatment centers should offer additional support to athletes, especially as they prepare for treatment discharge. Knowledgeable and experienced treatment staff are needed to comprehensively address the unique needs of athletes in intensive ED treatment (Sherman \& Thompson, 2001).

\section{Exercise in Intensive ED Treatment Centers}

Unbalanced exercise behavior is estimated to be present in more than $3 \%$ of the general population, $25 \%$ to $65 \%$ of athletes, and $33 \%$ to $100 \%$ of those with eating disorders (Reel \& Voelker, 2012). The negative ramifications resulting from prolonged compulsive exercise (i.e., fatigue, sleep disturbances, overuse injuries, difficulties with social relationships) can be especially destructive for athletes as health and sport performance are likely to deteriorate over time (Taranis \& Meyer, 2011). Since unbalanced or compulsive exercise is often a core feature of EDs, intensive treatment would seem to be an opportune time to comprehensively address exercise. Additionally, thoughts and behaviors related to unbalanced exercise (e.g., a belief that rest is unnecessary, exercise is solely utilized to alter appearance or to regulate emotional distress, excessive exercise beyond prescribed parameters) upon treatment discharge are among the strongest predictors of ED relapse (Carter, Blackmore, Sutander-Pinnock, \& Woodside, 2004). Yet intensive ED treatment facilities do not universally address exercise during treatment as intentionally and systematically as other ED treatment targets (e.g., decreasing body image distress and body distortions, increasing flexibility with food intake, etc.). Factors that might inhibit intensive ED treatment centers from systematically addressing exercise might include: an inability to come to a consensus, among staff, about the role of exercise in treatment (e.g., whether or not to permit patients to exercise in treatment); space constraints for fitness facilities; and an inability to identify knowledgeable and qualified staff who are sensitive and attuned to appropriately monitor and support exercise in treatment.

Although guidelines and outcomes specific to athletes in intensive ED treatment are limited, data have demonstrated the effectiveness of integrating exercise in treatment. Exercise has been shown to reinforce weight gain and increase body mass index (BMI) in inpatients with anorexia nervosa (AN) and supervised aerobic and strength training exercises have been shown to effectively reduce eating disorder symptoms (Calogero \& Pedrotty, 2004; Carei et al., 2010; Chantler, Szabo, \& Green, 2006; Sundgot-Borgen, Rosenvinge, Bahr, \& Schneider, 2002), depression (Carei et al., 2010; Sherman et al., 2005), and anxiety (Carei et al., 2010) in EDs. It is important to note that these studies have appropriately entailed individualized and supervised exercise in the context of 
treatment rather than independent and unsupervised exercise. Furthermore, Moola, Gairdner, and Amara (2013) found that nutritionally supported exercise lessens food preoccupation, enhances treatment compliance, increases bone density, and improves quality of life in medically stable inpatients with AN. Exercise has also been shown to increase muscle strength in patients with $\mathrm{AN}$, though no significant increases in BMI were found in these studies (del Valle et al., 2010; Fernandez-del-Valle et al., 2014). Additional measures of strength and BMI are needed to investigate the effectiveness of exercise within intensive ED treatment centers for all patients, particularly athlete patients.

\section{Current Study}

The present study investigates the effectiveness of incorporating exercise into intensive ED treatment for athletes and non-athletes. Specifically, the following questions were evaluated: 1) if all patients engaging in a graded exercise program improved in physical measures at discharge compared to admission; 2) if AN patients undergoing weight gain improved in physical measures at discharge compared to admission; and 3) if athletes and non-athletes differed in physical measures at admission and/or discharge.

\section{Methods}

\section{Participants}

The current study was part of a larger study examining outcomes within an intensive ED treatment program. Psychological measures from this larger study are reported elsewhere (Fewell, Levinson, \& Stark, 2017; Fewell, Nickols, Tierney, \& Levinson, 2018). A total of 21 male and female athlete patients and 36 male and female non-athlete patients who were enrolled in either residential or partial hospitalization treatment at an ED treatment facility between December 2012 and June 2017 were included in this study. Average length of stay was 65.75 days $(S D=26.22)$ for athletes and 67.75 days for non-athletes $(S D=28.31)$. Patients were diagnosed with the following EDs: AN (restricting or binge/purge subtype), bulimia nervosa (BN), other specified feeding or eating disorder (OSFED), bingeeating disorder (BED), or avoidant/restrictive food intake disorder (ARFID). Diagnoses were made by board certified psychiatrists upon treatment admission. Additionally, 13 patients $(22.8 \%)$ had a diagnosis of osteopenia and five patients $(8.7 \%)$ had a current physical injury or health issue. Please see Table 1 for a full description of patient characteristics.

\section{Treatment Program}

Treatments for both athlete and non-athlete groups used a combination of individual, family, and group therapies and included nutrition therapy, cognitive behavioral therapy, dialectical behavior therapy, exposure therapy (specifically at meal time), psychoeducation, and the experiential therapies (i.e., art therapy, dance/movement therapy). All patients met with a board-certified psychiatrist at 


\section{Table 1 Demographic and Clinical Characteristics of Athlete and Non-Athlete Patients}

\begin{tabular}{lcc}
\hline & Athlete $\boldsymbol{n = 2 1}$ & Non-Athlete $\boldsymbol{n}=\mathbf{3 6}$ \\
\hline Gender, $n(\%)$ & $17(81.0)$ & $31(86.1)$ \\
$\quad$ Female & $4(19.0)$ & $5(13.9)$ \\
$\quad$ Male & $21(100)$ & $33(91.7)$ \\
European American, $n(\%)$ & & \\
ED Diagnoses, $n(\%)$ & $14(66.7)$ & $26(72.2)$ \\
AN & $2(9.5)$ & $2(5.6)$ \\
BN & $3(14.3)$ & $5(13.9)$ \\
OSFED & $2(9.5)$ & $1(2.8)$ \\
BED & $0(0)$ & $1(2.8)$ \\
$\quad$ ARFID & $4(19)$ & $9(30)$ \\
Diagnosis of Osteopenia, $n(\%)$ & $3(14.3)$ & $2(5.6)$ \\
Injury or health issue, $n(\%)$ & $65.75(26.22)$ & $67.75(28.31)$ \\
LOS in days, $M(S D)$ & 16 to 107 days & 27 to 132 days \\
LOS range & $18.57(2.96)$ & $20.47(7.20)$ \\
Age in years, $M(S D)$ & 13 to 22 years & 12 to 46 years \\
Age range & $3.14(2.94)$ & $5.81(6.78)$ \\
Duration of ED in years, $M(S D)$ & 0 to 11 years & 0 to 34 years \\
Duration of ED range &
\end{tabular}

Note. AN $=$ Anorexia Nervosa BN $=$ Bulimia Nervosa OSFED = Other Specified Feeding or Eating Disorder; $\mathrm{BED}=$ Binge Eating Disorder; $\mathrm{ARFID}=$ Avoidant Restrictive Food Intake Disorder; LOS = Length of Stay in days for the total number of days in either residential and/or partial hospitalization programming.

least once per week and psychopharmacological treatments were implemented as needed and determined by a psychiatrist. Treatment for all patients also included a non-active group therapy on the psychology of exercise, which was modeled on recommendations from Reel (2015). This group therapy entailed psychoeducation about exercise, as well as discussions about exercise motivations, compulsive thoughts related to exercise, and ways to cultivate a more mindful approach to exercise.

In addition to the therapies listed above, all patients participated in individual and group fitness sessions as part of ED treatment. The individual and group fitness sessions included circuit training; daily walks at a low intensity (approximately 30 minutes in duration); weekly modified, restorative yoga groups at a low intensity; recreational fitness outings (e.g., hiking, kayaking, Zumba, etc.); and weekly open gym with supported supervision from a National Strength and Conditioning Association (NSCA) certified strength and conditioning specialist (CSCS). Sessions with the CSCS included discussing specific goals before the session, instruction and supportive dialogue throughout the session, debriefing post-session on challenges and experiences during the session, and developing 
future goals after the session. The CSCS closely collaborated with patients' treatment teams to determine medical and clinical appropriateness to integrate fitness into treatment. The CSCS compiled individualized exercise prescriptions (e.g., the mode, duration, intensity, and frequency of physical activity) for patients and communicated with the registered dietitian (RD) to ensure each patient's energy expenditure was nutritionally supported by their meal plan.

Participation in fitness sessions were contingent upon clearance from a board certified primary care physician whereby patients were deemed to be medically stable. Additionally, patients needed to be compliant in treatment (e.g., adherent to meal plan, participating in individual and group therapy, etc.) and demonstrate psychological readiness before fitness was approved. Specifically, the patient's treatment team evaluated the following criteria to warrant psychological readiness to begin fitness: the ability to self-regulate excessive movement/standing; acceptance of meal plan increases to support exercise; utilization of a variety of additional coping behaviors to self-sooth or regulate emotion; and decreased perseveration related to exercise.

Fitness sessions, groups, and outings were accompanied by the CSCS to support patients as they were exposed to various fitness activities that were deliberately selected by the CSCS to maintain a safe and non-judgmental fitness environment. If the activity included an outside instructor (e.g., not an employee of the treatment program), the CSCS consulted with the instructor prior to the fitness outing about specialized considerations for the patients to uphold the fitness activity's intent to be body-positive and supportive. Importantly, sensitivity was given to potential challenges and triggers that may occur with exposure to fitness environments (e.g., body checking in mirrors in a group exercise studio, challenges with feeling fatigued in introductory sessions that can be a catalyst for negative-self talk or comparison). The CSCS provided support to those patients who experienced challenges specific to body image, comparisons, and other challenges that often accompany the process in working to develop a more balanced relationship to fitness in eating disorder treatment.

\section{Recommendations on Specialized ED Treatment for Athletes}

In addition to the standard ED treatment, athlete patients received group therapies on topics such as sport psychology, sport nutrition, psychology of exercise, body image in sport and life, family systems (sport family and family of origin), athlete process groups, and an additional weekly fitness group. Program staff members had expertise in treating both athletes and eating disorders (e.g., sport psychologist, sports dietitian, CSCS) and were sensitive to the athlete's identity as an athlete as well as the challenges associated with having an ED in sport. Furthermore, athlete patients received individualized sport specific training with a CSCS, which included an exercise prescription intended to develop autonomy throughout treatment. Exercise and sport specific training were tailored to individual patient needs, and the duration and intensity of sessions progressively increased in accordance with weight and therapeutic indicators. Finally, athlete patients had the option to include sport personnel (i.e., coaches, sports medicine doctor, strength and conditioning coach, athletic trainer, etc.) during treatment to 
collaborate with the ED treatment team and assist with facilitating the return to sport after discharging from treatment.

\section{Key Program Characteristics}

The program recognized the value of including physical education and balanced fitness training practices into treatment. The addition of supported physical activity to the ED treatment model is believed to be beneficial to recovery and overall health once an individual is medically stable and demonstrates an ability to follow their meal plan and consistently refrain from eating disorder and compulsive exercise behaviors. The program facilitated physical education in the overall treatment model to allow patients the opportunity to gain awareness and challenge cognitions and mindset associated with unbalanced exercise behaviors. Patients frequently processed thoughts and feelings around exercise with the CSCS throughout treatment, and they participated in supported individual and group fitness sessions with the CSCS to reshape their relationship with fitness.

The program understood the importance of including an eating disorder informed and sensitive fitness professional (NSCA-certified strength and conditioning specialist) to address harmful fitness training practices that the patientathletes were accustomed to. In an effort to help patient-athletes learn how to engage in fitness in a healthy and balanced manner, program staff provided structure, in-vivo experiences, and psychoeducation specific to: tuning into individual body cues, mental skills training for sport through sport psychology groups, individual sessions with a sport psychologist, proper sports nutrition education with a sports dietitian (CSSD) and the importance of rest and training recovery days. As a result of offering patient-athletes the opportunity to gain greater autonomy and independence through following and independent exercise prescription during treatment, patient-athletes were often more confident and adept to consistently care for themselves upon discharging from treatment.

The program encouraged patients to develop greater respect toward their bodies while improving awareness to the body's needs through holistic selfcare and the use of proper form, function, and biodynamics when exercising. The CSCS and staff helped patients engage in "intuitive exercise" whereby patients developed a mindful and attentive approach to be more attuned to the body's cues so intensity could be modified appropriately (Reel \& Voelker, 2012). Compulsive and avoidant exercise habits were addressed during the treatment process and patients were given opportunities and skills to practice alternative, adaptive coping behaviors when experiencing a compulsive urge to exercise or when desiring to avoid physical activity altogether. The program also included therapies focused on selfacceptance to reduce judging and comparing mindsets that fuel the critical overvaluation on body size and shape.

\section{Procedure}

Upon medical clearance from a board certified primary care physician, the following physical measures were obtained near treatment admission and discharge: maximal oxygen consumption, vertical jump, grip strength, and push-ups. Body mass index was also obtained at admission and discharge. This study was 
approved by the Institutional Review Board at Washington University in St. Louis prior to data collection.

\section{Physical Outcome Measures}

Body Mass Index (BMI) was assessed using a medical grade Detecto precision scale and height tool at both treatment admission and discharge by an approved staff. Patients were weighed in light clothing and were not informed of their weight.

Maximal Oxygen Consumption $\left(\mathrm{VO}_{2} \mathrm{max}\right)$ measures the amount of oxygen used during intensive exercise and is considered an excellent indicator of cardiovascular and aerobic endurance ("VO2 Max Testing", 2017). To obtain patients' $\mathrm{VO}_{2}$ max data, male patients were instructed to perform 24 complete cycles and female patients to perform 22 complete cycles of an "up, up, down, down" rhythm on a twelve-inch step for three minutes. The cycle was regulated with a metronome set at 96 beats per minute for males and 88 beats per minute for females. Upon completion of the three-minute cycle, the CSCS measured patients' recovery heart rate (HR) for 15 seconds and multiplied by four to obtain heart beats per minute. The CSCS then used the following equations to calculate patients' $\mathrm{VO}_{2}$ max scores: $\mathrm{VO}_{2} \mathrm{max}=111.33-(0.42 \times \mathrm{HR})$ for males; $\mathrm{VO}_{2} \mathrm{max}=65.81-(0.1847 \times \mathrm{HR})$ for females.

Vertical jump was utilized to assess patients' power output and was measured at the treatment center using the Just Jump measuring device by Vertical Systems ("Just Jump", 2015). Patients were instructed to stand with feet together and arms lifted above their heads while on the mat. Patients then were instructed to jump as high as possible, and the Just Jump device calculated patients' vertical jump by computing the length of time that patients were off the mat. Patients were instructed to jump two separate times and the highest value was recorded.

Grip Strength was utilized as a measure of isometric hand and forearm muscle strength. Using a Smedley III Analog Grip Strength Dynamometer ("Strength Training", n.d.), patients were instructed to grip the device with maximal effort three separate times and the highest value of the three was retained.

Push-ups were totaled in number and recorded as a measure of muscular endurance of the upper body. Male patients completed push-ups in the standard high plank position with legs straight and arms shoulder width apart. Female patients completed modified push-ups on the hands and knees with hands shoulderwidth apart and elbows fully extended. All patients completed as many repetitions as possible without breaking form. Scores from patients who required additional modifications (i.e., wall push-up positions) were excluded from this study.

\section{Analyses}

Paired sample t-tests were conducted for each outcome measure to examine change over time from treatment admission to treatment discharge in the full sample. Next, we used independent t-tests to compare athletes and non-athletes on each outcome measure at both treatment admission and treatment discharge. Finally, we tested if each outcome measure changed from treatment admission to discharge in patients with AN only using paired sample t-tests. 


\section{Results}

As shown in Table 2, patients in the full sample significantly improved their total number of push-ups at discharge $(M=21.29 ; S D=13.14)$ compared to admission $(M=15.15 ; S D=10.97, \mathrm{t}(54)=-8.89, p<.001$; see Table 2$)$. Upon discharge, grip strength scores in both the right $\left(M_{\text {right }}=29.21, S D=7.11\right)$ and left $\left(M_{\text {left }}=28.09\right.$, $S D=7.93)$ hands were significantly higher compared to admission $\left(M_{\text {right }}=27.86\right.$, $\left.S D=8.27 ; M_{l e f t}=26.98, S D=8.50, p s<.05\right)$. Similarly, number of push-ups and grip strength scores in both the right and left hands were significantly higher upon discharge compared to admission in patients with AN only ( $p \mathrm{~s}<.001$; see Table 3 ). Maximal oxygen consumption and vertical jump did not significantly improve upon discharge in the full sample or in AN only, though scores did not significantly decrease in either sample ( $p$ s $>.40$; see Tables 2 and 3). Body mass index in patients with AN was significantly higher at discharge $(M=21.44, S D=2.05)$ compared to admission $(M=17.58, S D=2.78, \mathrm{t}(38)=-11.98, p<.001)$, with a 3.86 average increase in patients with $\mathrm{AN}(n=39, S D=2.01)$.

Athletes completed significantly more push-ups upon admission $(M=22.71$, $S D=11.64)$ than non-athletes $(M=11.06, S D=8.02, p<.001$; see Table 4$)$ and significantly more push-ups at discharge $(M=29.86, S D=13.61)$ than non-athletes

\section{Table 2 Paired Samples t-tests: $\mathrm{VO}_{2}$ max, Vertical Jump, Right and Left-Hand Grip Strength, and Push-Ups in the Full Sample}

\begin{tabular}{lccccc}
\hline & $\begin{array}{c}\text { Admission } \\
\boldsymbol{M}(\mathbf{S D})\end{array}$ & $\begin{array}{c}\text { Discharge } \\
\boldsymbol{M} \text { (SD) }\end{array}$ & $\boldsymbol{t}$ Value & sig. & $\boldsymbol{N}$ \\
\hline $\mathrm{VO}_{2}$ max & $47.10(7.37)$ & $44.97(22.74)$ & .772 & $p=.443$ & 57 \\
Vertical Jump & $10.33(16.38)$ & $8.47(22.30)$ & .86 & $p=.40$ & 55 \\
Right hand grip strength & $27.86(8.27)$ & $29.21(7.11)$ & -2.64 & $p=.011$ & 57 \\
Left hand grip strength & $26.98(8.50)$ & $28.09(7.93)$ & -2.72 & $p=.009$ & 57 \\
Number of push-ups & $15.15(10.97)$ & $21.29(13.14)$ & -8.89 & $p<.001$ & 55 \\
\hline
\end{tabular}

Note. $\mathrm{VO}_{2} \max =$ maximal oxygen consumption.

Table 3 Paired Samples t-tests in AN Patients: $\mathrm{BMI}, \mathrm{VO}_{2} \mathrm{max}$, Vertical Jump, Left and Right Hand Grip Strength, and Push-Ups

\begin{tabular}{lccccc}
\hline & $\begin{array}{c}\text { Admission } \\
\boldsymbol{M}(\boldsymbol{S D})\end{array}$ & $\begin{array}{c}\text { Discharge } \\
\boldsymbol{M}(\boldsymbol{S D})\end{array}$ & $\boldsymbol{t}$ Value & sig. & $\boldsymbol{N}$ \\
\hline BMI & $17.58(2.78)$ & $21.44(2.05)$ & -11.98 & $p<.001$ & 39 \\
$\mathrm{VO}_{2}$ max & $46.75(6.56)$ & $45.89(11.39)$ & .61 & $p=.545$ & 40 \\
Vertical Jump & $8.72(18.68)$ & $8.77(19.00)$ & -.09 & $p=.927$ & 55 \\
Right hand grip strength & $25.54(5.05)$ & $27.43(5.71)$ & -4.42 & $p<.001$ & 40 \\
Left hand grip strength & $24.61(5.53)$ & $26.20(5.92)$ & -3.96 & $p<.001$ & 40 \\
Number of push-ups & $14.97(10.65)$ & $20.97(12.99)$ & -7.96 & $p<.001$ & 39 \\
\hline
\end{tabular}

Note . $\mathrm{AN}=$ Anorexia Nervosa $; \mathrm{BMI}=$ body mass index; $\mathrm{VO}_{2} \mathrm{max}=$ maximal oxygen consumption . 


\section{Table 4 Independent Samples t-tests Comparing Athletes to Non-Athletes: $\mathrm{VO}_{2}$ max, Vertical Jump, Left and Right-Hand Grip Strength, and Push-Ups}

\begin{tabular}{lccccc}
\hline & Athlete (SD) & Non-Athlete (SD) & $\boldsymbol{t}$ & sig. & $\boldsymbol{N}$ \\
\hline Admission $\mathrm{VO}_{2} \max$ & $47.78(8.49)$ & $46.71(6.73)$ & -.53 & $p=.60$ & 56 \\
Discharge $\mathrm{VO}_{2} \max$ & $50.25(10.14)$ & $41.89(27.11)$ & -1.36 & $p=.18$ & 56 \\
Admission VJ & $9.39(25.27)$ & $11.08(7.00)$ & .38 & $p=.71$ & 56 \\
Discharge VJ & $3.41(34.67)$ & $11.60(7.66)$ & 1.33 & $p=.60$ & 54 \\
Admission RH grip & $28.64(8.76)$ & $27.40(8.05)$ & -.54 & $p=.60$ & 56 \\
Discharge RH grip & $30.52(7.39)$ & $28.44(6.93)$ & -1.07 & $p=.29$ & 56 \\
Admission LH grip & $28.90(9.89)$ & $25.86(7.49)$ & -1.31 & $p=.20$ & 56 \\
Discharge LH grip & $30.81(9.13)$ & $26.50(6.78)$ & -2.03 & $p=.05$ & 56 \\
Admission push-ups & $22.71(11.64)$ & $11.06(8.02)$ & -4.47 & $p<.001$ & 56 \\
Discharge push-ups & $29.86(13.61)$ & $16.00(9.74)$ & -4.40 & $p<.001$ & 54 \\
\hline
\end{tabular}

Note. $\mathrm{VO}_{2} \max =$ maximal oxygen consumption; $\mathrm{VJ}=$ vertical jump; $\mathrm{RH}$ grip $=$ right hand grip strength; LH grip = left hand grip strength.

$(M=16.00, S D=9.74, p<.001$; see Table 3$)$. Likewise, athletes had significantly higher grip strength scores in the left hand upon discharge $(M=30.81, S D=9.13)$ than non-athletes $(M=26.50, S D=6.78), p<.05)$. Left-hand grip strength upon admission and right-hand grip strength at admission and discharge did not differ between the two groups ( $p s>.20$; see Table 4). Maximal oxygen consumption and vertical jump did not significantly differ between groups at any time point ( $p$ s >.06; see Table 4).

\section{Discussion}

The aim of the current study was to examine the usefulness and appropriateness of incorporating exercise in both athlete and non-athlete treatments within an intensive ED treatment center. In support of previous research, patients demonstrated an increase in strength by performing significantly more push-ups and significantly stronger hand grip at discharge compared to admission. These findings held true in AN patients, despite an average BMI increase of nearly 4 units, demonstrating that strength was improved even during weight gain in AN. Maximal oxygen consumption and vertical jump did not significantly change from treatment admission to discharge, though this may be explained by the substantial increase in BMI and relatively short duration of ED treatment. Finally, athlete patients demonstrated a higher number of push-ups at treatment admission than non-athletes, as well as a higher number of push-ups and stronger left-hand grip upon discharge than non-athletes.

The realized strength improvements that athlete patients experienced throughout treatment are thought to help reinforce important treatment targets. Specifically, the strength gains could facilitate improved confidence in athlete patients' meal plans, acceptance of suggested goal weight, and adherence to fitness 
recommendations. Psychoeducation, providing rationale behind such treatment targets, was a component of athlete-patient's treatment. However, athlete-patients' experience of objective strength and power gains could be a powerful agent to support ongoing treatment and recovery.

In support of previous studies (Calogero \& Pedrotty, 2004; Carei et al., 2010; Chantler et al., 2006; Sundgot-Borgen et al., 2002), patients in the full sample demonstrated improvements in strength by increasing the number of push-ups and hand grip strength upon discharge. These improvements suggest that the introduction of strength training, in the context of supported supervision, proper fueling, and adequate rest and recovery periods, aided patients in the development of muscular neural adaptations in the early weeks of training (Baechle \& Earle, 2008). Additional research is needed to investigate if the continuation of proper fitness development practices lead to improved strength over time in patients recovering from EDs. Furthermore, studies could examine if patients are more committed and engaged in ED treatment or if body image changes occur when fitness is appropriately integrated and supported.

Patients with AN also demonstrated improved number of push-ups and hand grip strength upon treatment discharge, despite significant increases in BMI. Increased strength in $\mathrm{AN}$ is well supported by previous research (del Valle et al., 2010; Fernandez-del-Valle et al., 2014), but the concurrent increase in BMI while improving strength in $\mathrm{AN}$ is a relatively new finding. This is especially noteworthy given that complications of AN, such as endocrine and cardiorespiratory issues, gastrointestinal problems, and physical weakness can contribute to the deterioration of power and strength (Academy of Eating Disorders, n.d.). Given that these issues were present in the current sample and strength still improved, the findings suggest that increased nutritional intake, reduction in excessive exercise, or a combination of both remedied low energy availability and facilitated strength improvements.

In the full sample, $\mathrm{VO}_{2}$ max and vertical jump did not significantly differ from admission to discharge. $\mathrm{VO}_{2}$ max was measured via heart rate, however, which may have skewed the results. For instance, it is well documented that cardiovascular impairments such as bradycardia are prominent features of EDs, particularly in AN (Yahalom et al., 2013). Throughout ED treatment, it is expected for cardiovascular complications to improve and bradycardia to eventually resolve. However, an increased heart rate would cause an apparent negative impact on $\mathrm{VO}_{2}$ max scores upon treatment discharge, as the measured values were exclusively estimated through an equation using patients' recovery heart rates. Therefore, a calculated higher heart rate value would yield a lower $\mathrm{VO}_{2}$ max score.

The lack of significant findings for change in vertical jump is notable considering the substantial increase in BMI in the majority of the sample. Vertical jump may have also been impacted by the imposed limitations of intensive ED treatment and the relatively mild exercise regimes. For example, compulsive exercise is a common occurrence in EDs and contributes to the development and maintenance of EDs (Davis et al., 1997; Davis, Katzman, \& Kirsh, 1999). Compared to the excessive exercise many individuals engage in prior to seeking intensive ED treatment, decreased strength, in light of reduced exercise hours and intensity during ED treatment, could be assumed. Additionally, exercise may have been delayed upon treatment admission in patients with severe low weight or 
medical instability, resulting in less time engaging in fitness. Results suggest that patients may indeed be improving in strength measures, but these improvements are confounded by some of the therapeutic targets in ED treatment (i.e., weight gain and mild intensity exercise). Unsurprisingly, athletes had significantly greater number of push-ups than non-athletes at both admission and discharge and significantly stronger left-hand grip at discharge. Considering that athletes are typically required to dedicate a great deal of time and effort into sport training, these differences are sensible.

\section{Treatment Recommendations}

Medical stability and nutritional and behavioral compliance should be established before exercise regimes are put into place and then, once established, monitored on an ongoing basis. Safe and clinically appropriate exercise prescription is important and should take the following elements into consideration: level of care; vital signs and lab results; current fitness ability; adherence to daily meal plan and fluid requirements; achievement of weekly weight gain goals; amount of commitment to treatment targets; fitness goals and prior sport involvement; consistency in following fitness parameters; openness to be mindful and remain in the present moment during fitness sessions; and a willingness to listen and respond appropriately to body cues, pain, or discomfort.

While participating in fitness might initially heighten body image distress or compulsive exercise, adhering to a flexible and supportive fitness plan, coupled with following a balanced meal plan, typically lessens acute body image distress and compulsive exercise over time. Balanced fitness practices can help reduce body dissatisfaction when movement is introduced into treatment under the supervision of the multidisciplinary treatment team and a qualified and nonjudgmental fitness professional (i.e., the CSCS).

\section{Limitations}

Several limitations were present in the current study. First, although physical changes were captured, psychological changes, though likely, were not concurrently measured. Since the program's philosophy promotes the development of greater attunement with body cues and cultivating a more wholesome relationship with exercise, a measure such as the Intuitive Exercise Scale (IEXS; Reel, Galli, Miyairi, Voelker, \& Greenleaf, 2016) could be used to assess and better understand individuals' relationship with exercise. Second, time constraints often present in ED treatment centers may have led to limited displays of strength and power, since appropriate and safe fitness progression develops over time. Likewise, the inability to collect data on patients after treatment discharge serves as a limitation as it is unclear whether strength and power were maintained or further improved after discharge. Future research should examine whether maintenance or improvements in strength and power occur following ED treatment. Additionally, long-term ED prognosis following strength improvements should be examined, such as adherence to meal and exercise plans, weight and medical stabilization, and decreased ED cognitions.

Another limitation was the inability to accurately capture the degree of strength and power improvements for those patients who were unable to complete 
measures immediately upon treatment admission due to medical instability. Consequently, it is likely that these patients experienced more pronounced strength and power improvements than were observed. Future research could adopt modified versions of physical measures that would be safe and appropriate for all patients, regardless of medical status, to complete upon treatment admission to more accurately evaluate strength and power throughout treatment. As noted above, a limitation existed with the use of recovery heart rate in the $\mathrm{VO}_{2}$ max calculation; future studies should examine alternative methods to this calculation, such as using the $\mathrm{VO}_{2}$ max testing equipment. Finally, the non-homogenous nature of the patient population, such as mixed genders, ED diagnoses, and length of stay served as a limitation in the present study. Additional research is needed to investigate measures of strength and power in athletes and non-athletes engaged in intensive ED treatments across gender and ED diagnoses and with similar lengths of stay.

\section{Conclusion}

The findings in the current study suggest that the specialized exercise prescriptions utilized in the ED treatment center were effective to either increase or prevent reduction of strength in ED patients over the course of intensive ED treatment. Results suggest that improvements in athletic performance can occur when appropriate exercise and nutritional parameters are followed in ED treatment, especially for athletes who are concerned about sport performance decrements that might occur when seeking a higher level of ED treatment. Furthermore, since EDs and compulsive exercise are highly comorbid, ED treatment centers have the responsibility to address exercise pathology and to develop skills that support balanced exercise. Trained staff who are competent in treating EDs and who can incorporate fitness appropriately are vital to address the nuanced needs of athletes and compulsive exercise in ED treatment centers.

\section{References}

Academy of Eating Disorders. (n.d.). Position Statement: AED Emphasizes Physical Health more than BMI. Retrieved from http://www.aedweb.org/web/index.php/23-getinvolved/position-statements/158-aed-emphasizes-physical-health-more-than-bmi

Arthur-Cameselle, J.N., \& Quatromoni, P.A. (2014). Eating disorders in collegiate female athletes: Factors that assist recovery. Eating Disorders: The Journal of Treatment and Prevention, 22, 50-61. doi:10.1080/10640266.2014.857518

Baechle, T., \& Earle, R. (2008). Essentials of strength training and conditioning, 3, 94.

Bar, R.J, Cassin, S.E., \& Dionne, M.M. (2016). Eating disorder prevention initiatives for athletes: A review. European Journal of Sport Science, 16(3), 325-335. 25815432 doi: 10.1080/17461391.2015.1013995

Baum, A. (2006). Eating disorders in the male athlete. Sports Medicine, 36(1), 1-6. 16445307 doi:10.2165/00007256-200636010-00001

Becker, C.B., McDaniel, L., Bull, S., Powell, M., \& McIntyre, K. (2012). Can we reduce eating disorder risk factors in female college athletes? A randomized exploratory investigation of two peer-led interventions. Body Image, 9(1), 31-42. 22019502 doi: 10.1016/j.bodyim.2011.09.005 
Beumont, P.J.V., Arthur, B., Russell, J.D., \& Touyz, S.W. (1994). Excessive exercise in dieting disorder patients: Proposals for a supervised exercise program. International Journal of Eating Disorders, 15, 21-36. doi:10.1002/1098-108X(199401)15:1<21:: AID-EAT2260150104>3.0.CO;2-K

Bompa, T. (1983). Theory and methodology of training: The key to athletic performance. Toronto, Canada: Human Kinetics.

Calogero, R.M., \& Pedrotty, K.N. (2004). The practice and process of healthy exercise: An investigation of the treatment of exercise abuse in women with eating disorders. Eating Disorders: The Journal of Treatment and Prevention, 12(4), 273-291. doi:10.1080/ 10640260490521352

Carei, T.R., Fyfe-Johnson, A.L., Breuner, C.C., \& Brown, M.A. (2010). Randomized controlled clinical trial of yoga in the treatment of eating disorders. Journal of Adolescent Health, 46(4), 346-351. 20307823 doi:10.1016/j.jadohealth.2009.08.007

Carter, J.C., Blackmore, E., Sutander-Pinnock, K., \& Woodside, D.B. (2004). Relapse in anorexia nervosa: A survival analysis. Psychological Medicine, 34, 671-679. 15099421 doi:10.1017/S0033291703001168

Chantler, I., Szabo, C.P., \& Green, K. (2006). Muscular strength changes in hospitalized anorexic patients after an eight week resistance training program. International Journal of Sports Medicine, 27, 660-665. 16874594 doi:10.1055/s-2005-865812

Cook, B.J., Wonderlich, S.A., Mitchell, J.E., Thompson, R., Sherman, R., \& McCallum, K. (2016). Exercise in eating disorders treatment: Systematic review and proposal of guidelines. Medicine \& Science in Sports \& Exercise, 48, 1408-1414. 26909533 doi: 10.1249/MSS.0000000000000912

Davis, C., Katzman, D.K., Kaptein, S., Kirsh, C., Brewer, H., Kalmbach, K., . . Kaplan, AS. (1997). The prevalence of high-level exercise in the eating disorders: Etiological implications. Comprehensive Psychiatry, 38, 321-326. 9406737 doi:10.1016/S0010440X(97)90927-5

Davis, C., Katzman, D.K., \& Kirsh, C. (1999). Compulsive exercise in adolescents with anorexia nervosa: A psychobehavioral spiral of pathology. Journal of Nervous and Mental Disease, 187, 336-342. 10379720 doi:10.1097/00005053-199906000-00002

del Valle, M.F., Pérez, M., Santana-Sosa, E., Fiuza-Luces, C., Bustamante-Ara, N., Gallardo, C., . . . Lucía, A. (2010). Does resistance training improve the functional capacity and well being of very young anorexic patients? A randomized controlled trial. Journal of Adolescent Health, 46(4), 352-358. 20307824 doi:10.1016/j.jadohealth. 2009.09.001

Fernandez-del-Valle, M., Larumbe-Zabala, E., Villaseñor-Montarroso, A., Cardona Gonzalez, C., Diez-Vega, I., Lopez Mojares, L.M., \& Perez Ruiz, M. (2014). Resistance training enhances muscular performance in patients with anorexia nervosa: A randomized controlled trial. International Journal of Eating Disorders, 47(6), 601-609. 24810684 doi:10.1002/eat.22251

Fewell, L.K., Levinson, C.A., \& Stark, S. (2017). Depression, worry, and psychosocial functioning predict eating disorder treatment outcomes in a residential and partial hospitalization setting. Eating and Weight Disorders, Studies on Anorexia, Bulimia, and Obesity, 4(4), 1-11. doi:10.1007/s40519-016-0357-6

Fewell, L.K., Nickols, R., Schlitzer Tierney, A., \& Levinson, C.A. (2018). Eating disorders in sport: Comparing athletes to non-athletes at an intensive eating disorder treatment center. Manuscript submitted for publication.

Greenleaf, C., Petrie, T.A., Carter, J., \& Reel, J.J. (2009). Female collegiate athletes: Prevalence of eating disorders and disordered eating behaviors. Journal of American College Health, 57(5), 489-495. 19254889 doi:10.3200/JACH.57.5.489-496

Harris, E.C., \& Barraclough, B. (1998). Excess mortality of mental disorder. British Journal of Psychiatry, 173(1), 11-53. doi:10.1192/bjp.173.1.11 
Joy, E., De Souza, M.J., Nattiv, A., Misra, M., Williams, N., Mallinson, R.J., . . . Expert Panel. (2014). Female athlete triad coalition consensus statement on treatment and return to play of the female athlete triad: 1st International Conference held in San Francisco, California, May 2012 and 2nd International Conference held in Indianapolis, Indiana, May 2013. British Journal of Sports Medicine, 48(4), 289. doi:10.1136/bjsports-2013-093389

Joy, E., Kussman, A., \& Nattiv, A. (2016). 2016 update on eating disorders in athletes: A comprehensive narrative review with a focus on clinical assessment and management. British Journal of Sports Medicine, 50, 154-162. 26782763 doi:10.1136/bjsports2015-095735

Just Jump. (2015). Retrieved from http://www.power-systems.com/p-3839-just-jump.aspx Katz, J. (1996). Clinical observations on the exercise of anorexia nervosa. In W.F. Epling \& W.D. Pierce (Eds.), Activity anorexia: Theory, research, and treatment (pp. 199-207). Mahwah, NJ: Lawrence Erlbaum.

Martinsen, M., Bahr, R., Børresen, R., Holme, I., Pensgaard, A.M., \& Sundgot-Borgen, J. (2014). Preventing eating disorders among young elite athletes: A randomized controlled trial. Medicine \& Science in Sports \& Exercise, 46, 435-447. 24549033 doi:10.1249/MSS.0b013e3182a702fc

Moola, F.J., Gairdner, S.E., \& Amara, C.E. (2013). Exercise in the care of patients with anorexia nervosa: a systematic review of the literature. Mental Health and Physical Activity, 6(2), 59-68.

Mountjoy, M., Sundgot-Borgen, J., Burke, L., Carter, S., Constantini, N., Lebrun, C., . . Ljungqvist, A. (2014). The IOC consensus statement: Beyond the female athlete triad-Relative energy deficiency in sport (RED-S). British Journal of Sports Medicine, 48, 491-497. 24620037 doi:10.1136/bjsports-2014-093502

Olmsted, M.P., Woodside, D.B., Carter, J.C., McFarlane, T.L., Staab, R., Colton, P.A., \& Molleken, L.L. (2007). Intensive treatments for eating disorders. In G.O. Gabbard (Ed.), Treatments of psychiatric disorders (4th ed., pp. 709-722). Washington, DC: American Psychiatric Press.

Plateau, C.R., Arcelus, J., Leung, N., \& Meyer, C. (2017). Female athlete experiences of seeking and receiving treatment for an eating disorder. Eating disorders, 25(3), 273-277. 28051927 doi:10.1080/10640266.2016.1269551

Reel, J.J. (2015). The relationship between exercise and eating disorders: A double-edged sword. In M.B. Andersen \& S.J. Hanrahan (Eds.), Doing exercise psychology. Champaign, IL: Human Kinetics.

Reel, J.J., Galli, N., Miyairi, M., Voelker, D., \& Greenleaf, C. (2016). Development and validation of the intuitive exercise scale. Eating Behaviors, 22, 129-132. 27289049 doi:10.1016/j.eatbeh.2016.06.013

Reel, J.J., \& Voelker, D. (2012). Exercise to the extreme? Identifying and addressing unhealthy exercise behaviors. In R. Schinke (Ed.), Athletic Insight's writings of 2012 (pp. 301-315). Hauppauge, NY: Nova Science.

Sherman, K.J., Cherkin, D.C., Erro, J., Miglioretti, D.L., \& Deyo, R.A. (2005). Comparing yoga, exercise, and a self-care book for chronic low back pain. Annals of Internal Medicine, 143, 849-856. 16365466 doi:10.7326/0003-4819-143-12-20051220000003

Sherman, R.T., \& Thompson, R.A. (2001). Athletes and disordered eating: Four major issues for the professional psychologist. Professional Psychology-Research \& Practice, 32(1), 27-33. 29684829 doi:10.1037/0735-7028.32.1.27

Strength Training. (n.d.). Retrieved from https://www.chponline.com/store/cart.php

Sundgot-Borgen, J. (1993). Prevalence of eating disorders in elite female athletes. International Journal of Sport Nutrition, 3(1), 29-40. 8499936 doi:10.1123/ijsn. 3.1 .29 
Sundgot-Borgen, J., Rosenvinge, J.H., Bahr, R., \& Schneider, L.S. (2002). The effect of exercise, cognitive therapy, and nutritional counseling in treating bulimia nervosa. Medicine \& Science in Sports \& Exercise, 34(2), 190-195. 11828224 doi:10.1097/ 00005768-200202000-00002

Sundgot-Borgen, J., \& Torstveit, M.K. (2004). Prevalence of eating disorders in elite athletes is higher than in the general population. Clinical Journal of Sport Medicine, 14(1), 25-32. 14712163 doi:10.1097/00042752-200401000-00005

Taranis, L., \& Meyer, C. (2011). Associations between specific components of compulsive exercise and eating-disordered cognitions and behaviors among young women. International Journal of Eating Disorders, 44, 452-458. 20721896 doi:10.1002/ eat. 20838

Torres-McGehee, T.M., Green, J.M., Leaver-Dunn, D., Leeper, J.D., Bishop, P.A., \& Richardson, M.T. (2011). Attitude and knowledge changes in collegiate dancers following a short-term, team-centred prevention program on eating disorders. Perceptual and Motor skills, 112, 711-725. 21853760 doi:10.2466/06.PMS.112.3. 711-725

VO2 Max Testing. (2017). Retrieved from https://med.virginia.edu/exercise-physiologycore-laboratory/fitness-assessment-for-community-members-2/vo2-max-testing/

Yahalom, M., Spitz, M., Sandler, L., Heno, N., Roguin, N., \& Turgeman, Y. (2013). The significance of bradycardia in anorexia nervosa. International Journal of Angiology, 22(2), 83-94. 24436590 doi:10.1055/s-0033-1334138 